SECTION 25. Technologies of materials for the light and textile industry.

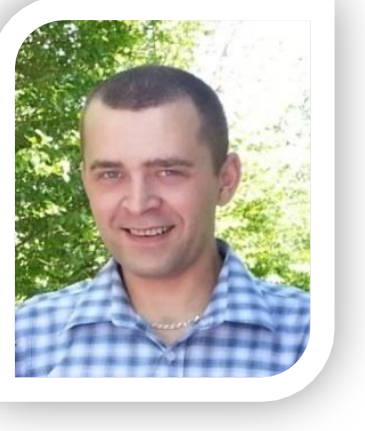

\author{
Shevtsov Alexandr Nikolayevich \\ candidate of technical Sciences, associate Professor of \\ the Department «Applied mathematics» \\ Taraz State University named after M.Kh. Dulati,
} Kazakhstan Abzalbekov Bekzhan
doctoral student of the Department «Technology of
textile industry»
Taraz State University named after M.Kh. Dulati,
Kazakhstan
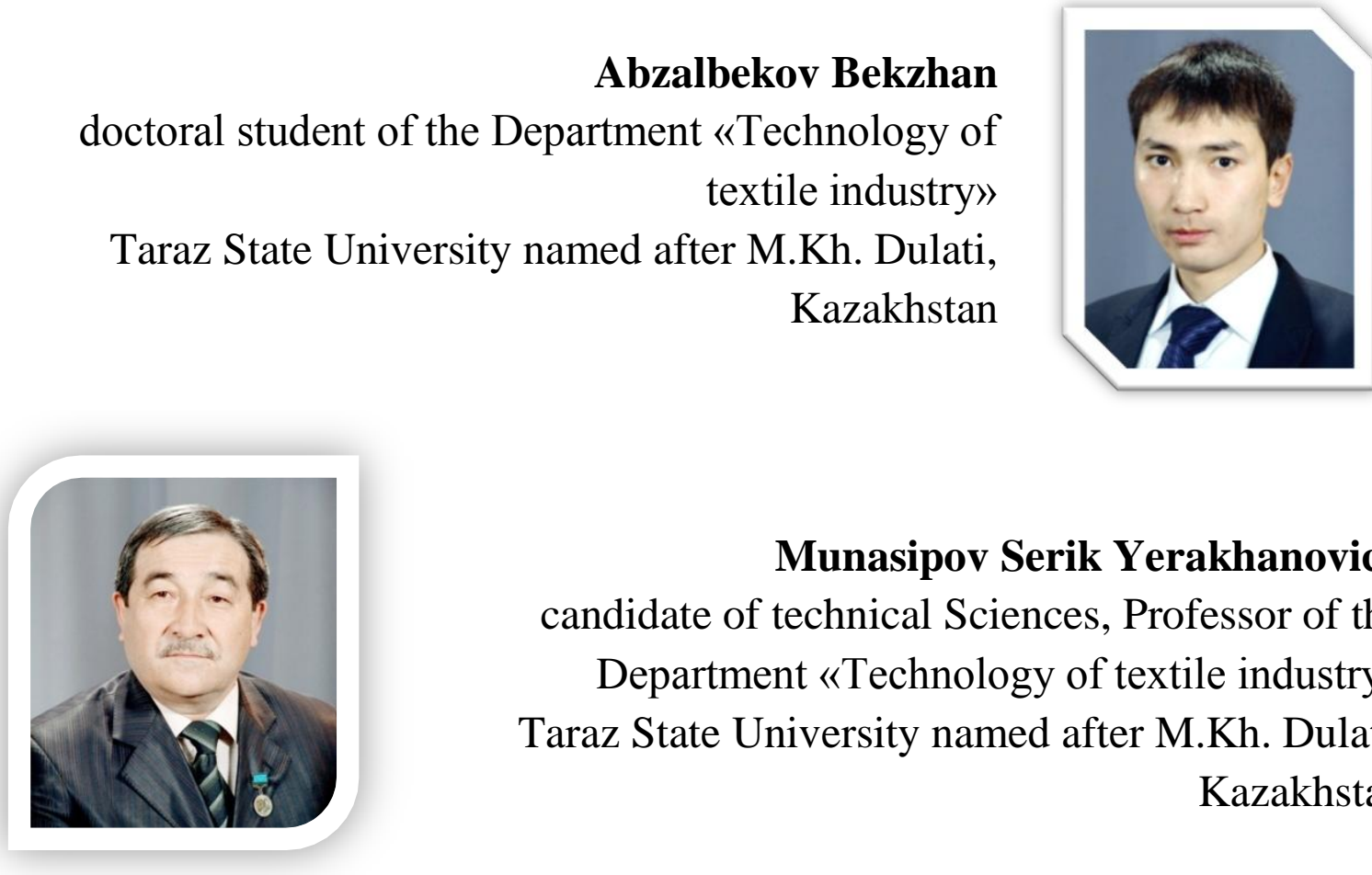

Munasipov Serik Yerakhanovich candidate of technical Sciences, Professor of the

Department «Technology of textile industry» Taraz State University named after M.Kh. Dulati, Kazakhstan

\title{
CONSTRUCTION OF MATHEMATICAL MODELS OF ORTHOPEDIC INSOLES FOR SHOES PATIENTS WITH DIABETES MELLITUS.
}

In this article the model is developed orthopedic insoles on the basis of the conducted research the feet of patients with diabetes mellitus..

Key words: diabetes, insole, shoes, mathematical model.

\section{ПОСТРОЕНИЕ МАТЕМАТИЧЕСКОЙ МОДЕЛИ ОРТОПЕДИЧЕСКИХ СТЕЛЕК ДЛЯ ОБУВИ БОЛЬНЫХ САХАРНЫМ ДИАБЕТОМ.}

В данной статье разрабатывается модель ортопедической стельки на основе проведенных исследований стоп больных сахарным диабетом.

Ключевые слова: диабет, стелька, обувь, математическая модель. 
При разработке рациональных конструкций обуви любого назначения и отдельных ее деталей большое значение имеют антропометрические данные о форме и размерах стоп [1]. Поэтому, для определения рациональных параметров ортопедических стелек были проведены антропометрические исследования стоп больных диабетом [2].

В результате проведенных исследований больных диабетом и другими заболеваниями приводящими к уменьшению кровотока в нижних конечностях, выявлена необходимость периодического массажа стопы и в особенности пальцев. Для этой цели целесообразно использовать именно ортопедическую стельку определенной формы и различной толщины в разных местах. При выборе формы и толщины и межстелечных слоев наиболее важной характеристикой материала является относительная остаточная деформация при сжатии, а также необходимо учитывать антропометрические особенности стопы, возраст, вес человека.

Стелька изготовленная из полимерных материалов, приобретает способность к упругой деформации сжатия и в данном случае, играет роль амортизатора, позволяющего сгладить пики динамических нагрузок при ходьбе, беге и прыжках, а определенный рельеф поверхности стельки может оказывать необходимый терапевтический массажный эффект.

Для улучшения эксплуатационных свойств ортопедических стелек можно использовать материалы с высокой степенью деформации при сжатии, что обеспечит более мягкий терапевтический эффект и увеличит комфортность использования ортопедических стелек.

С этой целью разработаем математическую модель и компьютерную программу для моделирования рельефа стельки. В начале определим опорные точки для модели (рис.1). В качестве материала для проведения исследования у нас имеется 500 фотографий стоп больных диабетом (рис.2). 


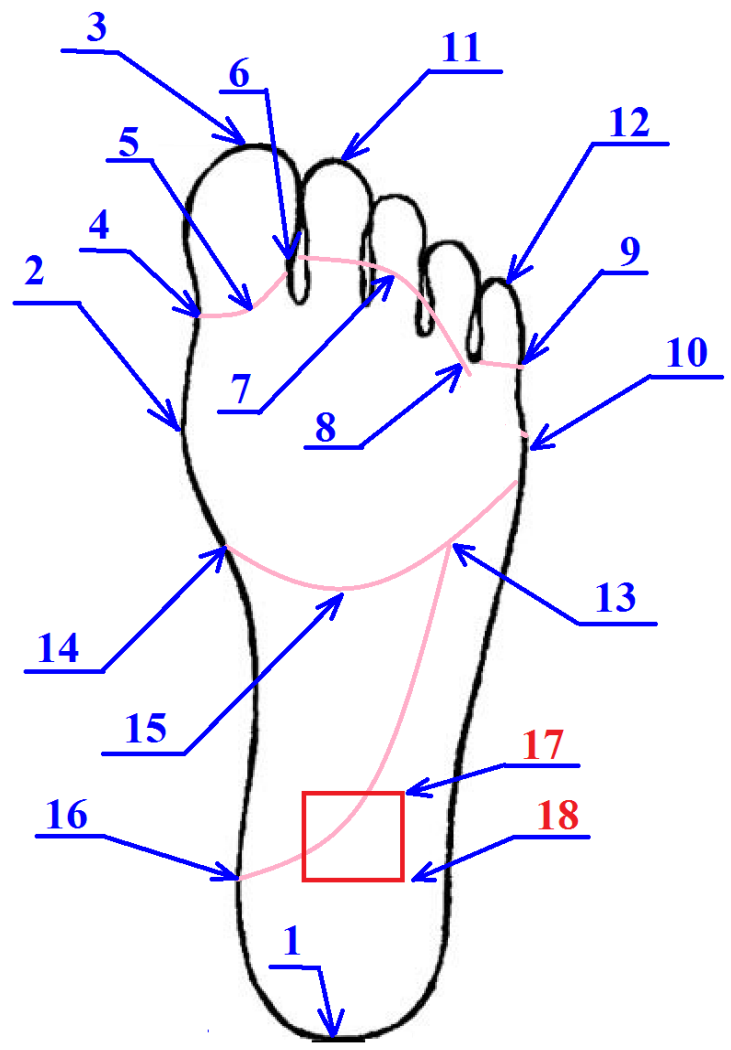

Рисунок 1 - Нумерация опорных точек.
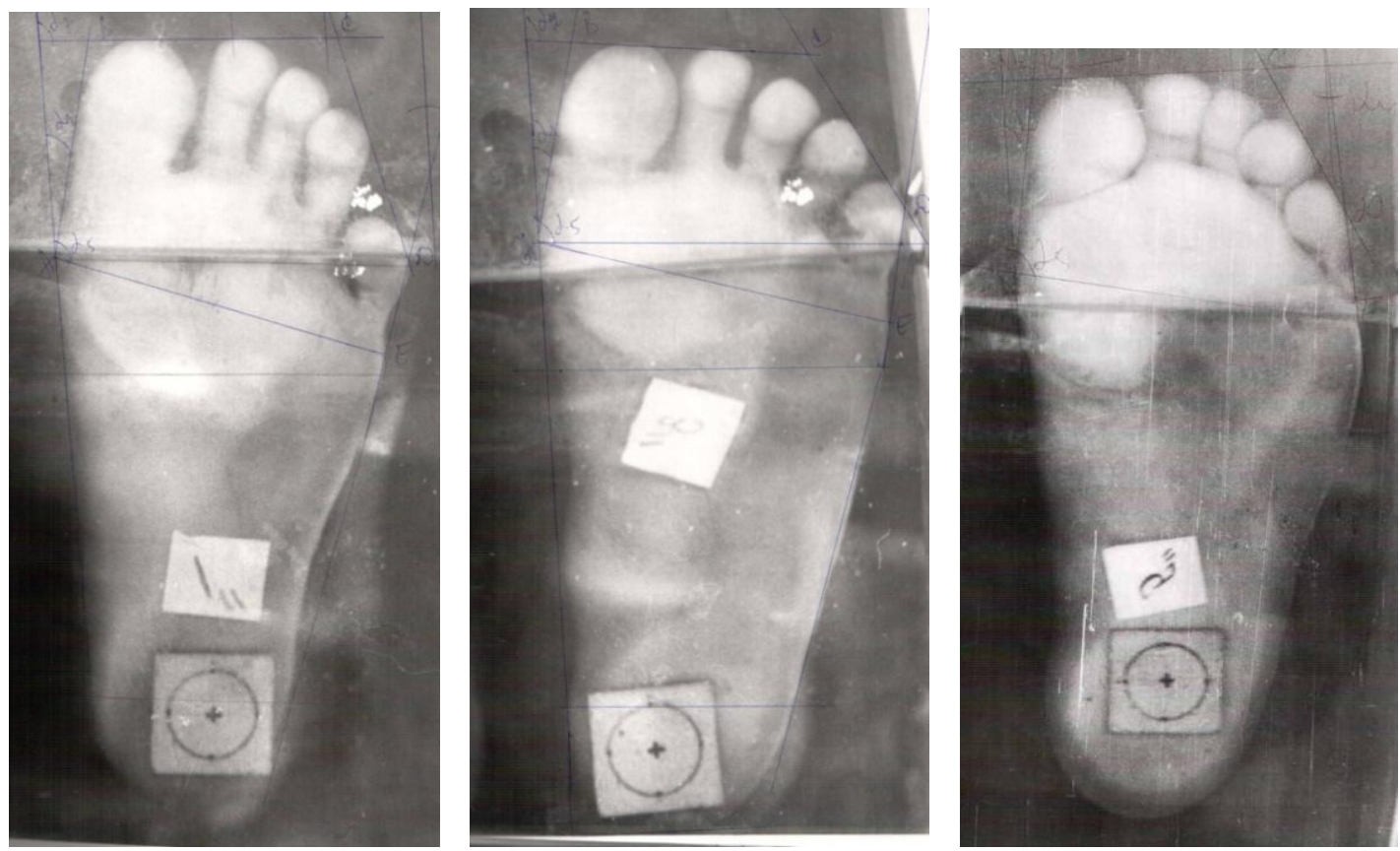

Рисунок 2 - Фотографии стоп с метрическим квадратом. 


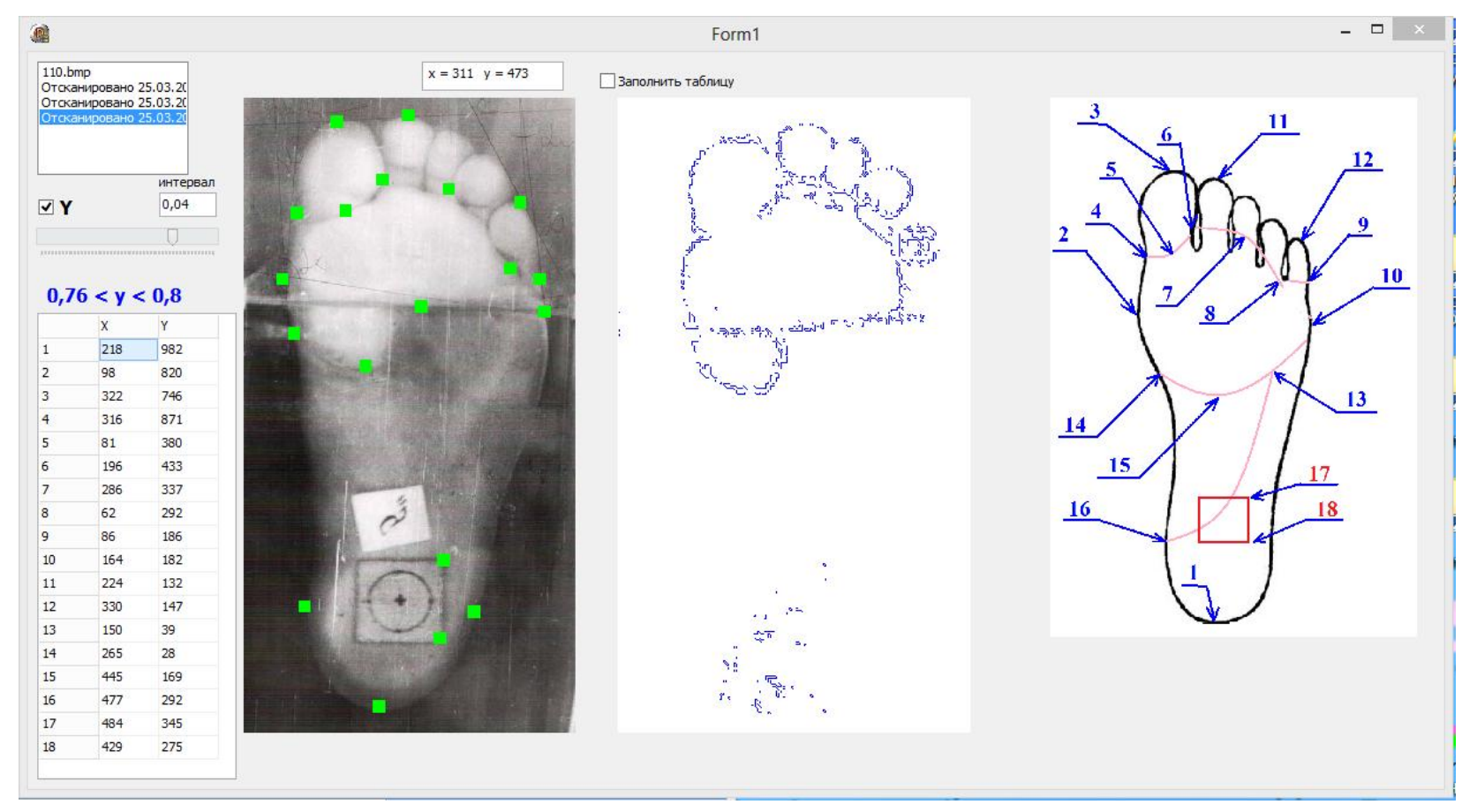

\section{Рисунок 3 -Разработанная программа.}

Разработаем программу для анализа фотографий и получения антропометрических данных (рис.3). Программа позволяет быстро выделить опорные точки математической модели, занести их в таблицу и сохранить все данные в отдельный файл, для каждого рисунка стопы, для дальнейших исследований. Для расширения функциональности программы в нее встроен яркостный анализатор изображения и выделения определенного пользователем участка спектра в формате $\mathrm{YCbCr}$. Подобрав необходимый спектр, можно увидеть зоны повышенных нагрузок на стопу, и зоны минимальных нагрузок.

Для определения формы массажных дуг, между пальцев, можно разбить дугу на две дуги, или воспользоваться интерполяционным многочленом. Рассмотрим разбиение на две отдельные дуги точкой «6», как видно из (рис.3, рис.4). Введем следующие обозначения: точки А и В центры окружностей; точки C, D, E - края массажных дуг; R1 и R2 радиусы окружностей. 

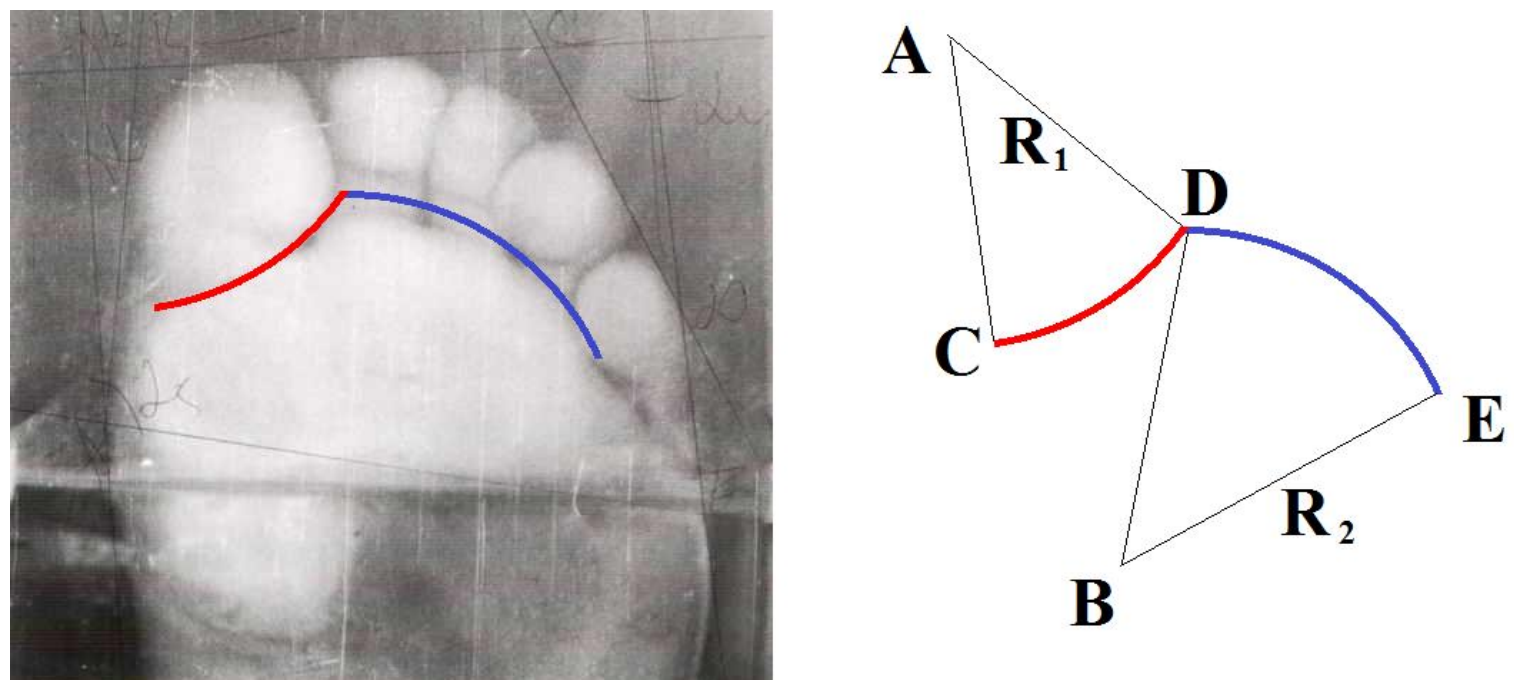

Рисунок 4 - Формы массажных дуг.

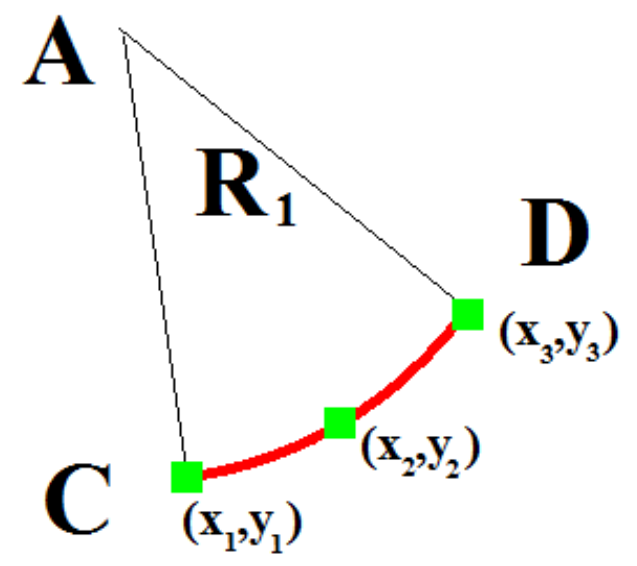

\section{Рисунок 5 - Координаты точек дуги.}

Зная координаты трех точек дуги (рис.5) можно определить ее параметры, координаты центров и радиусы.

Тогда, решая полученную систему (отдельно для каждой дуги)

$$
\left\{\begin{array}{l}
\left(x_{1}-x\right)^{2}+\left(y_{1}-y\right)^{2}=R^{2} \\
\left(x_{2}-x\right)^{2}+\left(y_{2}-y\right)^{2}=R^{2} \\
\left(x_{3}-x\right)^{2}+\left(y_{3}-y\right)^{2}=R^{2}
\end{array}\right.
$$

в среде компьютерной алгебры Maple, получим следующие значения координат центра окружности: 


$$
\begin{aligned}
& x=\frac{1}{2} \frac{\left(x_{1}^{2}\left(y_{2}-y_{3}\right)+x_{2}{ }^{2}\left(-y_{1}+y_{3}\right)+x_{3}{ }^{2}\left(y_{1}-y_{2}\right)+y_{1}{ }^{2}\left(y_{2}-y_{3}\right)+y_{2}{ }^{2}\left(y_{3}-y_{1}\right)+y_{3}{ }^{2}\left(y_{1}-y_{2}\right)\right)}{\left(x_{1}\left(y_{2}-y_{3}\right)+x_{2}\left(y_{3}-y_{1}\right)+x_{3}\left(y_{1}-y_{2}\right)\right)}, \\
& y=-\frac{1}{2} \frac{\left(x_{1}^{2}\left(x_{2}-x_{3}\right)+x_{2}{ }^{2}\left(-x_{1}+x_{3}\right)+x_{3}{ }^{2}\left(x_{1}-x_{2}\right)+y_{1}{ }^{2}\left(x_{2}-x_{3}\right)+y_{2}{ }^{2}\left(x_{3}-x_{1}\right)+y_{3}{ }^{2}\left(x_{1}-x_{2}\right)\right)}{\left(x_{1}\left(y_{2}-y_{3}\right)+x_{2}\left(y_{3}-y_{1}\right)+x_{3}\left(y_{1}-y_{2}\right)\right)} .
\end{aligned}
$$

Для рассчета будем использовать тройки чисел $(4,5,6)$ и $(6,7,8)$. Для нахождения радиусов, достаточно найти расстояние от любой из трех точек до центра окружности.

$$
R=\sqrt{\left(x_{1}-x\right)^{2}+\left(y_{1}-y\right)^{2}} .
$$

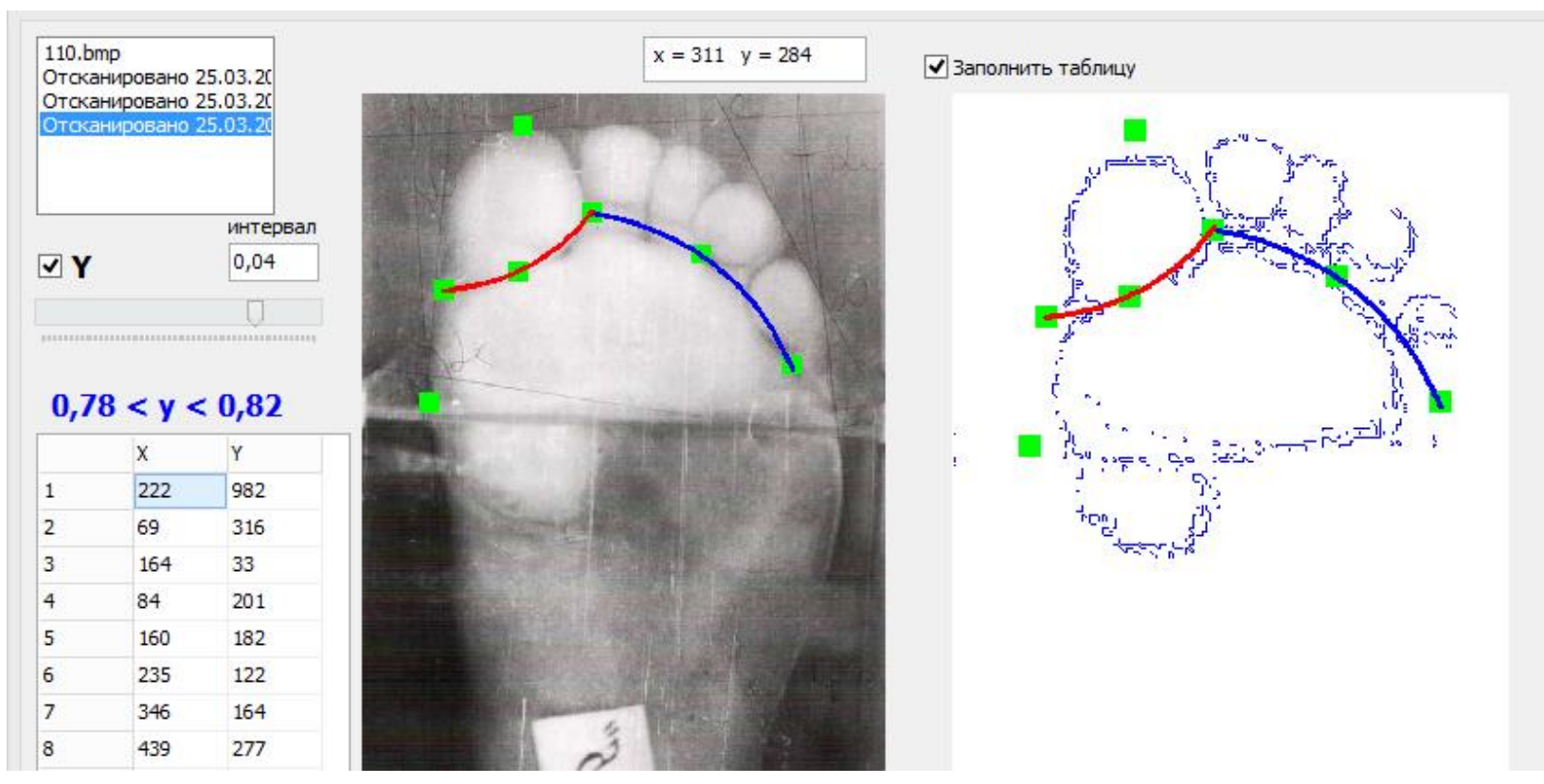

Рисунок 6 - Массажные дуги

Код программы для рассчета дуг, запишем в следующем виде:

code:

function duga(x1,y1,x2,y2,x3,y3:integer;color:Tcolor;tr:boolean): integer; var $\mathrm{x}, \mathrm{y}, \mathrm{r}:$ integer;

I: Integer;

begin

$\mathrm{x}:=\operatorname{trunc}((\mathrm{x} 1 * \mathrm{x} 1 *(\mathrm{y} 2-\mathrm{y} 3)+\mathrm{x} 2 * \mathrm{x} 2 *(-\mathrm{y} 1+\mathrm{y} 3)+\mathrm{x} 3 * \mathrm{x} 3 *(\mathrm{y} 1-\mathrm{y} 2)+\mathrm{y} 1 * \mathrm{y} 1 *(\mathrm{y} 2-$

$\mathrm{y} 3)+\mathrm{y} 2 * \mathrm{y} 2 *(\mathrm{y} 3-\mathrm{y} 1)+\mathrm{y} 3 * \mathrm{y} 3 *(\mathrm{y} 1-\mathrm{y} 2)) /$

$(\mathrm{x} 1 *(\mathrm{y} 2-\mathrm{y} 3)+\mathrm{x} 2 *(\mathrm{y} 3-\mathrm{y} 1)+\mathrm{x} 3 *(\mathrm{y} 1-\mathrm{y} 2)) / 2)$;

$\mathrm{y}:=-\operatorname{trunc}((\mathrm{x} 1 * \mathrm{x} 1 *(\mathrm{x} 2-\mathrm{x} 3)+\mathrm{x} 2 * \mathrm{x} 2 *(-\mathrm{x} 1+\mathrm{x} 3)+\mathrm{x} 3 * \mathrm{x} 3 *(\mathrm{x} 1-\mathrm{x} 2)+\mathrm{y} 1 * \mathrm{y} 1 *(\mathrm{x} 2-$

$\mathrm{x} 3)+\mathrm{y} 2 * \mathrm{y} 2 *(\mathrm{x} 3-\mathrm{x} 1)+\mathrm{y} 3 * \mathrm{y} 3 *(\mathrm{x} 1-\mathrm{x} 2)) /$ 


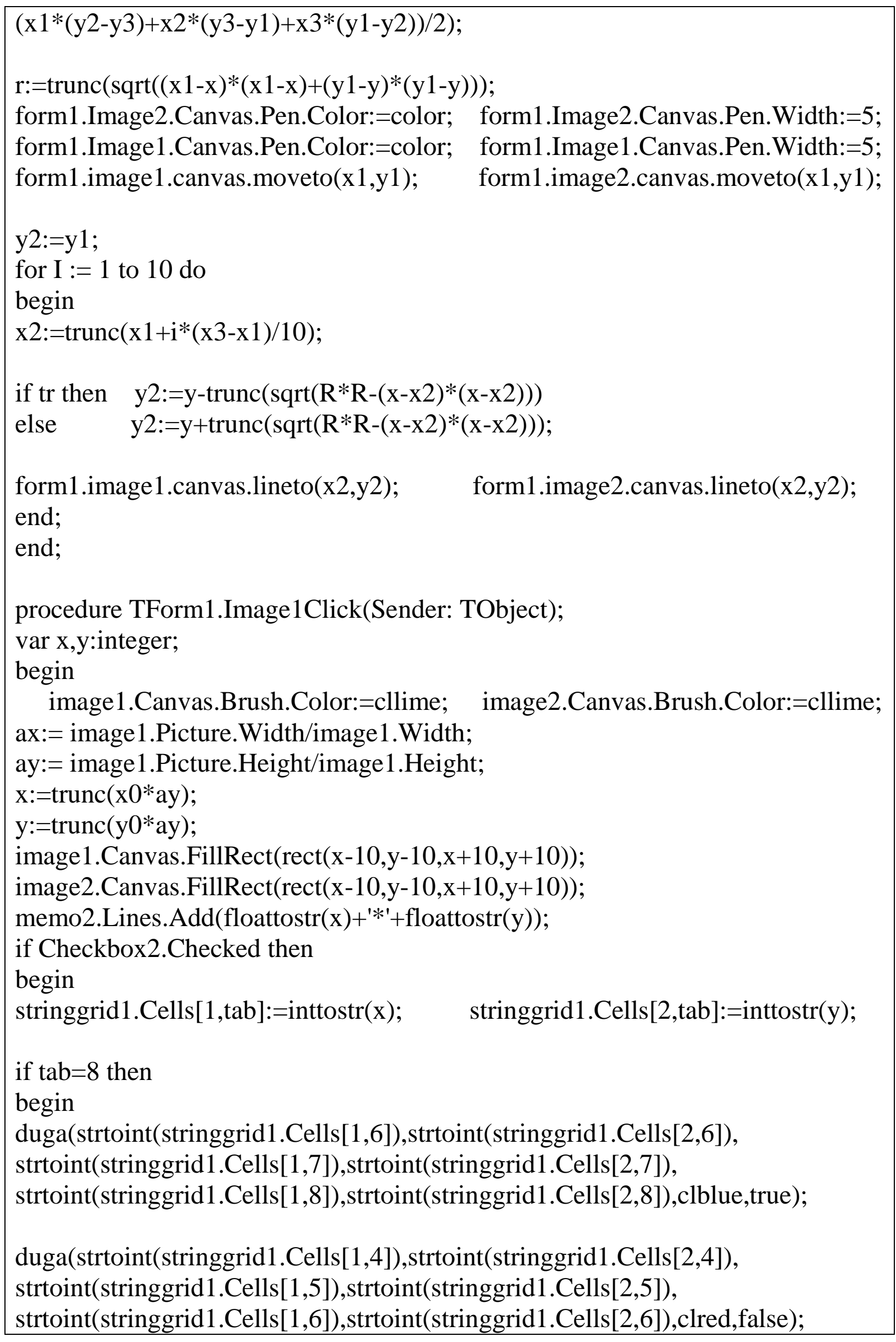


end;

inc(tab);

if $t a b>18$ then

begin

Checkbox2.Checked:=false;

memo2.Lines.SaveToFile(fname+'.txt');

end; end;

end;

По результатам проведенного исследования, можно сделать следующие выводы:

- нами проведены общирные антропометрические исследования стоп больных сахарным диабетом,

- получены фотоплантограммы стоп больных диабетом,

- при анализе плантограмм получены координаты опорных точек плантарной поверхности стопы,

- разработана программа для анализа фотографий и антропометрических данных плантарной поверхности стопы,

- результаты исследовании будут способствовать разработке оптимальных параметров ортопедических стелек для обуви больных сахарным диабетом.

\section{Литература.}

1. Кочеткова Т.С., КлючниковаВ.М. Антропологические и биомеханические основы конструирования изделий из кожи. М., Легпромбытиздат, 1991.

2. Абзалбекулы Б., Мунасипов С.Е., Джумабекова Г.Б. Джанахметов О.К. Антропометрические исследования стоп больных сахарным диабетом. МН-ПК «Технологии и управление: проблемы, идеи, инновации». Филиал ФГБОУ ВПО «Московский государственный университет технологий и управления им.К.Г.Разумовского». г.Тверь. 29 апреля 2013 г. 